\title{
New drugs in respiratory disorders: II
}

\author{
D C FLENLEY
}

In this second article I will discuss the newer antimicrobial drugs and compare them with established antimicrobial drugs.

\section{New antimicrobial agents \\ TRIMETHOPRIM}

Trimethoprim, a selective antagonist of bacterial dihydrofolate reductase, is combined with sulphamethoxazole in cotrimoxazole, which is of proved value in lower respiratory tract infections, in particular the mixed infection of Haemophilus influenzae and Streptococcus pneumoniae that occurs so commonly in patients with chronic bronchitis and emphysema. ${ }^{1}$ A comparison of trimethoprim alone with co-trimoxazole showed no difference in outcome in adults with bronchitis, chronic bronchitis, or pneumonia, ${ }^{2}$ but side effects (rashes, nausea, etc) were more common with co-trimoxazole. Trimethoprim is marginally cheaper than co-trimoxazole and should therefore be considered when treatment with co-trimoxazole is indicated. Co-trimoxazole in high doses remains the treatment of choice, however, in infection with Pneumocystis carinii in immune compromised patients. ${ }^{3}$

\section{AMOXYCILLIN WITH CLAVULANIC ACID (AUGMENTIN)}

$\beta$-lactamases, which open the $\beta$-lactam ring of penicillins and cephalosporins, are a major source of bacterial resistance to these antibiotics. ${ }^{4}$ The combination of amoxycillin with a $\beta$-lactamase inhibitor, clavulanic acid, as in Augmentin (250 mg amoxycillin $+125 \mathrm{mg}$ clavulanic acid per tablet) is effective against many such respiratory pathogens, including most ampicillin resistant strains of $H$ influenzae. ${ }^{5}$ " Augmentin was therefore effective in $97 \%$ of patients with pneumonia, bronchiectasis, and bronchitis, ${ }^{7}$ but pathogens were identified in only $25 \%$ of these patients, even when transtracheal aspiration was used, and $\beta$-lactamase producing organisms were not important. Minor gastrointestinal disturbances or rashes occurred in $30 \%$ of patients. ${ }^{6}$ Only $6 \%$ of strains of $H$ influenzae currently isolated in the United Kingdom, however, are ampicillin resistant. ${ }^{8}$ Thus first line treatment with Augmentin for an acute exacerbation of chronic bronchitis scarcely seems justifiable in preference to amoxycillin or ampicillin alone as Augmentin costs half as much again as the same dose of amoxycillin alone.

Department of Respiratory Medicine, University of Edinburgh, City Hospital, Edinburgh EH10 5SB

D C FLENLEY, PHD, FRCP, professor of respiratory medicine

\section{CEPHALOSPORINS}

Of the 11 cephalosporins or cephamycins listed in the 1982 British National Formulary, ${ }^{9}$ only cephalexin, cephradine, and cefaclor are active when given by mouth, whereas the others (cefotaxime, cefoxitin, cefuroxime, cefsulodin, cephaloridine, cephalothin, cephamandole, and cephazolin) must be given parenterally. What is the role for this plethora of cephalosporin antibiotic drugs in today's respiratory medicine? Unfortunately, the results that are available from trials rarely give a definitive answer, but the evidence will be briefly discussed for four major areas of bacterial infection of the lower respiratory tractpneumonia acquired outside hospital; acute exacerbation of chronic bronchitis and emphysema; pneumonia in a previously compromised host; and chronic infections (cystic fibrosis, bronchiectasis, etc). The more general use of cephalosporins is considered elsewhere.

The new cephalosporins have few side effects. Pain after intramuscular injection is rare but may be reduced by adding lignocaine. The drugs are mostly excreted unchanged by both glomerular filtration and tubular secretion, so that renal failure may lead to toxic concentrations. Probenecid will prolong the half life. Hypersensitivity rashes may occur in $2-5 \%$ of all recipients and cross reactivity may occur in $5-16 \%$ of patients known to be sensitive to penicillin. The combination of a cephalosporin-for instance, cephaloridine-with an aminoglycoside may be nephrotoxic. ${ }^{10}$

\section{Pneumonia acquired outside hospital}

Pneumonia acquired outside hospital is still most often due to Strep pneumoniae, ${ }^{11}$ which is nearly always as sensitive to penicillin as to any of the current cephalosporins. ${ }^{12}$ There is thus no justification for using a cephalosporin in preference to penicillin in treating proved Strep pneumoniae pneumonia ${ }^{13}$ except when the particular organism is known to be resistant to penicillin. $^{14}$

\section{Chronic bronchitis and emphysema}

In chronic bronchitis and emphysema Strep pneumoniae and $H$ influenzae are the commonest pathogens in the sputum. ${ }^{1}$ Of the oral cephalosporins, only cefaclor is active against both. ${ }^{12} \mathrm{~A}$ 10 day course of cefaclor ( $250 \mathrm{mg}$ three times a day), however, is three times more expensive than an equivalent course of ampicillin. ${ }^{15}$ There was no difference in clinical outcome in a comparative trial of these two drugs in 50 patients aged 65-85, but the causative pathogens were not determined. ${ }^{16}$ Furthermore, although $250 \mathrm{mg}$ cefaclor three times daily "cured" $93 \%$ of patients with pneumonia or acute exacerbations of chronic bronchitis ( $97 \%$ with $500 \mathrm{mg}$ twice daily ${ }^{17}$ ), the results of another trial showed $250 \mathrm{mg}$ of ampicillin three times a day for seven days to be as effective in an acute exacerbation of chronic 
bronchitis as either $500 \mathrm{mg}$ of ampicillin or amoxycillin in either 250 or $500 \mathrm{mg}$ doses, all given four times daily. ${ }^{18}$

Therefore in treating purulent exacerbations of chronic bronchitis and emphysema there seems little to justify oral cefaclor in preference to oral ampicillin (or amoxycillin), and clearly cephalexin and cephradine (the other drugs active when given by mouth) are not indicated as they are not active against both Strep pneumoniae and $H$ influenzae.12 If, however, the patient has a proved infection with an ampicillin resistant strain of $H$ influenzae cefuroxime, ${ }^{19}$ cefotaxime, ${ }^{20}$ or ceftazidime ${ }^{21}$ are all active, at least in the laboratory. None the less, superior clinical efficacy, as compared with co-trimoxazole, ${ }^{22}$ or possibly ampicillin, ${ }^{23}$ remains to be established in a properly controlled clinical trial where the invading pathogens are identified before treatment. Although purulent exacerbations in patients with relatively mild chronic bronchitis may not be appreciably influenced by antibiotic treatment (tetracycline), ${ }^{24}$ severe exacerbations with $\mathrm{CO}_{2}$ retention (type 11 respiratory failure) still carry a mortality rate of $9-24 \% .{ }^{25} 26$

\section{Bacterial pneumonia in the compromised host}

Whether bacterial pneumonia in the compromised host results from a congenital abnormality-for example, hypogammaglobulinaemia-or is today more commonly induced therapeutically (as in organ transplantation, treatment of autoimmune disease, or malignancy) it is often due to Strep pneumoniae infection but may also arise from $H$ influenzae, Klebsiella spp, Pseudomonas aeruginosa, or anaerobic organisms. Klebsiella pneumonia carries a mortality of over $40 \%$, and although gentamicin is often an agent of first choice, in Britain, where chloramphenicol is rarely used in treating other conditions, this drug in combination with streptomycin and tetracycline has often been effective. Cefotaxime is very active against Klebsiella spp in vitro, as is cephamandole and other parenteral cephalosporins. ${ }^{12}$ Cefotaxime and ceftazidime have the greatest in vitro activity against Klebsiella spp. With cefotaxime this has been confirmed clinically, klebsiellae being eliminated in $91 \%$ of 189 adult patients, ${ }^{20}$ but the cases of pneumonia were not separately identified. Cefotaxime may be the only drug that is active against gentamicin resistant strains of Klebsiella spp. ${ }^{27}$ Mycobacterium tuberculosis, Legionella pneumophila, viral or fungal agents, and Pneumocystis carinii are other infective agents recognised as causing pneumonia in the compromised patient, ${ }^{28}$ but none will respond to any of the cephalosporin antibiotics. ${ }^{29}{ }^{30}$ Antiviral and antifungal chemotherapy are considered elsewhere in this series.

\section{Chronic infections}

Chronic infections include bronchiectasis, where again Strep pneumoniae and $H$ influenzae are common infective agents, with, more rarely, pseudomonas or anaerobes, or both, and cystic fibrosis, where in childhood staphylococcal infections and in the adult pseudomonas infections are common, particularly with the mucoid strain of Ps aeruginosa, which carries a particularly grave prognosis. ${ }^{31}$ Carbenicillin and gentamicin by aerosol over many months can slow the progression of cystic fibrosis in adults with pseudomonas. ${ }^{32}$ Cefsulodin and ceftazidime (not yet available in the United Kingdom) also seem very promising as they may be more active than any other cephalosporin against Ps aeruginosa. Ceftazidime is also active against pseudomonas strains resistant to carbenicillin. ${ }^{21 R}$

The cephalosporins are the most expensive of any group of antibiotics in use. Good patient care may be maintained despite a voluntary restriction of prescribing cephalosporins in hospital. ${ }^{33}$ Treatment with $2 \mathrm{~g}$ of cefotaxime a day for seven days costs $£ 90-£ 120$ as compared with $£ 1-£ 2$ for $250 \mathrm{mg}$ of ampicillin three times daily for seven days. ${ }^{9}$ Thus if all new episodes of an exacerbation of chronic bronchitis in the United Kingdom were treated with cefotaxime, which is certainly not recommended, $\underline{\underline{T}}$ the bill would be around $£ 100-£ 200 \mathrm{~m}$ a year for this alone 3 compared with $£ 1-2 m$ a year if ampicillin were used; these $\stackrel{\mathbb{Q}}{\varrho}$ figures were calculated from estimates of the probable numbers $c$ of such acute exacerbations each year in the United Kingdom. ${ }^{34} \widehat{\widehat{\Omega}}$ Ampicillin, amoxycillin, and co-trimoxazole (or possibly tri- $\overline{\bar{J}}$ methoprim alone) are rational choices for first line treatment of this very common condition, so allowing excellent patient care to $\mathbb{\infty}^{\circ}$ be combined with sensible thrift.

\section{Erythromycin}

Erythromycin may have a new role as possibly the most effective drug for treating legionnaires' disease, in addition to its $\widehat{\nabla}$ traditional role as an alternative to penicillin for use in penicillin sensitive patients and also for treating chlamydial pneumonia in infancy. ${ }^{12}$ Although evidence from a controlled trial is not $\vec{\circ}$ available, in infection with $L$ pneumophilia (legionnaires' disease, $\vec{\overrightarrow{ }}$ pneumonia, and bacteraemia, Pontiac fever, and febrile illness ${ }_{\mathscr{\sigma}}$ without pneumonia) erythromycin, possibly at first given $\frac{\partial}{\partial}$ intravenously in the sick patient followed by oral maintenance 3 . treatment in a dose of $2-4 \mathrm{~g} /$ day for three weeks, is recommended. $\mathbb{\infty}_{\infty}$ Relapse has been noted with shorter courses of treatment. Rifampicin is also effective in vitro but should not be used as the $\mathcal{E}$ sole antimicrobial drug. $L$ pneumophilia produces a $\beta$-lactamase, of which may explain the lack of effect of penicillin and cephalo- of sporins. ${ }^{35}$ Erythromycin estolate is well absorbed by mouth but ${ }^{\mathcal{N}}$ may cause liver damage, whereas erythromycin stearate and 9 propionate both yield lower serum concentrations, but this does $\vec{\varphi}$ not seem to affect the results of treatment, and neither of these 3 preparations is hepatotoxic. Intravenous erythromycin is given as the lactobionate, $0.5 \mathrm{~g}$ every six hours for adults. In severe $\stackrel{\rho}{\mathcal{S}}$ infections this may be increased to $6-8 \mathrm{~g}$ daily, ${ }^{36}$ but intra- $\vec{\varphi}$ muscular injection is not advisable.

\section{Anaerobic pulmonary infections}

\section{METRONIDAZOLE}

Metronidazole is active against nearly all Gram negative $\overrightarrow{\vec{O}}$ anaerobic bacteria - that is, the anaerobes that contribute to the 3 mixed flora of lung abscess ${ }^{37}$ and aspiration pneumonia and most strains of Bacteroides fragilis. Metronidazole by mouth $(500 \mathrm{mg}$. four times daily in an adult) yields adequate serum concentrations at much less cost than the very expensive intravenous form. Serious neurological side effects (vertigo, ataxia, confusion, and fits) or reversible peripheral neuropathy are rare at these doses, 3 . except with prolonged courses or in patients with liver disease ${ }^{38}$ Penicillin, ampicillin, and amoxycillin are all also active against many anaerobes (except for $\beta$ lactamase producing Bacteroides spp) as are most cephalosporins.

\section{CLINDAMYCIN}

Clindamycin is active against many anaerobes, including penicillin resistant strains, and is thus superior to penicillin in treating lung abscess, ${ }^{38}$ being actively transported to the 0 infection site in leucocytes. It causes diarrhoea in $10-20 \%$ of $\frac{D}{\Phi}$ patients which usually improves when the drug is stopped, but $\stackrel{\mathcal{P}}{+}$ may cause Clostridium difficile induced colitis in $0 \cdot 1-10 \%$ of patients. $^{38}$

\section{Influenza}

AMANTADINE

Amantadine, which has an established use in the management

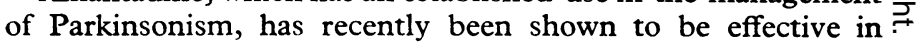


preventing influenza $\mathrm{A}$ infection during an epidemic when used in a dose of $100 \mathrm{mg}$ twice daily in the adult. ${ }^{39}$ Side effects of insomnia, jitteriness, and lack of concentration occurred in $13 \%$ of these patients but quickly cleared when the drug was stopped. Although yearly prophylactic immunisation with influenza vaccine remains the recommended policy for those patients who are at risk, particularly those with chronic pulmonary, cardiac, or renal disease, amantadine may also be useful in treating established influenza $\mathrm{A}$ infection as there is rapid relief of symptoms and thus return to work. ${ }^{40}$

\section{Chemotherapy for tuberculosis}

\section{RIFAMPICIN}

Rifampicin has radically changed the drug treatment of tuberculosis. The former 18-24 month regimens using streptomycin, isoniazid, and aminosalicylic acid (now designated classic chemotherapy) have been replaced in economically developed countries by standard chemotherapy-rifampicin $(600 \mathrm{mg}$ if the patient is over $50 \mathrm{~kg}, 450 \mathrm{mg}(8-12 \mathrm{mg} / \mathrm{kg})$ for those under $50 \mathrm{~kg})$, given with isoniazid $300 \mathrm{mg}(5 \mathrm{mg} / \mathrm{kg})$ once daily in a combination tablet of both drugs taken one hour before breakfast on an empty stomach for nine months. In addition, ethambutol $(15 \mathrm{mg} / \mathrm{kg})$ is included for the first two months. This standard chemotherapy has been shown to be effective, with insignificant relapse rates, in controlled trials in Britain ${ }^{41}{ }^{42}$ and elsewhere.

Rifampicin is fully absorbed, with therapeutic concentrations maintained for 12 hours due to an enterohepatic recirculation after an oral dose. Most of the drug is excreted in the bile and with daily treatment minor skin reactions, gastric upsets, and a rise in liver transaminases are all recognised but uncommon side effects, particularly during the early weeks of treatment, but these resolve despite continuing treatment. Thrombocytopenia, probably due to a circulating antibody, is a rare but important side effect as it is a contraindication to further use of the drug, but the platelet count rises when treatment is stopped. Clinical hepatitis is uncommon with today's standard chemotherapy, but pre-existing severe liver disease remains as a possible contraindication to treatment with rifampicin.

Rifampicin induces liver enzymes, so increasing the elimination of common drugs such as warfarin, corticosteroids, and sulphonylureas; whose dosage will thus need to be increased during treatment with rifampicin. Oral contraceptives are also metabolised more rapidly, and an alternative contraceptive method should be used during treatment with rifampicin. Intermittent rifampicin treatment, which is not included in the standard regimen, can induce a flu-like syndrome, with fever and chills, after three months' treatment because of a circulating antibody. If the drug is restarted after a long interval the dose should be built up gradually. Rifampicin is contraindicated in pregnancy. An intravenous rifampicin preparation is available for those too ill to take tablets. ${ }^{43}$

\section{ETHAMBUTOL}

Ethambutol is included in the standard regimen to prevent development of rifampicin resistance if the patient's organisms are initially resistant to isoniazid. This occurred in under $2 \%$ of all cases in the United Kingdom in 1978-9 but in $8 \%$ of cases originating in the Indian subcontinent. ${ }^{44}$ It is only a weak antituberculosis drug, $80 \%$ of the drug being absorbed after an oral dose, with a plasma half life of some eight hours. Optic neuritis, indicated by blurred vision, loss of visual acuity, and inability to see colours, is a recognised hazard in doses over $25 \mathrm{mg} / \mathrm{kg}$ but is rare if the dose is not above $15 \mathrm{mg} / \mathrm{kg}$, as in the standard chemotherapy regimen. Vision usually returns after stopping the drug, but patients should be warned to report any visual disturbance during treatment with ethambutol.

\section{PYRAZINAMIDE}

Pyrazinamide, introduced in 1954, was previously considered too hepatotoxic for wide use. Interest has been reawakened for use in lower doses as a valuable part in short courses of antituberculosis regimens, possibly by killing mycobacteria within macrophages at a low intracellular $\mathrm{pH}$ and so reducing the relapse rate in six month regimens. ${ }^{45} 46$ Thus an oral daily dose of $1.5 \mathrm{~g}$ (for those weighing under $50 \mathrm{~kg}$ ) to $2.5 \mathrm{~g}$ (weight over $75 \mathrm{~kg}$ ) for two months, in combination with rifampicin and isoniazid, showed no excess hepatotoxicity (as detected by liver function tests, gastrointestinal symptoms, or jaundice) that could be attributed to the pyrazinamide alone. ${ }^{47}$ Pyrazinamide has a role in the management of tuberculous meningitis as it rapidly diffuses into the cerebrospinal fluid. Rifampicin, isoniazid (10-12 $\mathrm{mg} / \mathrm{kg}$ intramuscularly or intravenously), pyrazinamide $30 \mathrm{mg} / \mathrm{kg}$ to a maximum of $2.5 \mathrm{~g}$ daily, and streptomycin $1.0 \mathrm{~g}$ daily (or $0.75 \mathrm{~g}$ daily if the patient is over 35) are combined, along with pyridoxine $50 \mathrm{mg}$ daily by mouth to prevent the risk of peripheral neuropathy from the high dose of isoniazid. Corticosteroids are also used to prevent formation of adhesions and hydrocephalus in treating this life threatening condition.

\section{SHORT COURSE CHEMOTHERAPY}

In patients whose sputum is positive on smear there are now several effective regimens lasting for six months, based on rifampicin, isoniazid, and pyrazinamide for the first two months (some including thrice weekly streptomycin in addition) followed by isoniazid and rifampicin for the remaining four months. ${ }^{46}$ Even shorter regimens are being evolved. ${ }^{47}$

\section{COMPLIANCE WITH TREATMENT}

Compliance with treatment is now the main problem remaining in managing tuberculosis. Both the patients and all their doctors must realise that cure can be guaranteed only if all doses are taken for the whole course of treatment. This advice must be repeated frequently. The reddish colour of the urine after taking rifampicin can be intensified by extraction with butanol, so proving that the drug has been taken within 12-24 hours. Supervised chemotherapy may be needed in feckless patients, which includes alcoholics and drug addicts. Partially treated patients may excrete drug resistant organisms and so be a menace to themselves and to others. ${ }^{4}$ ?

\section{Cytotoxic chemotherapy}

Chemotherapy in treating lung cancer has attracted much recent interest. Cytotoxic agents in combination are now being widely used in small cell lung cancer, where they may offer a substantial improvement in survival over that to be expected without treatment, although cure or prolonged survival is possible in very few. ${ }^{48}$ These drugs are thus used only in inoperable cases, which in small cell cancer unfortunately includes most cases, as the disease is usually disseminated at the time of diagnosis. The most active agents include cyclophosphamide, doxorubicin, vincristine, methotrexate, lomustine, and etoposide. Although all are active when used alone, combining these drugs in various regimens gives the best results. The drugs are usually given parenterally either together or only a day or two apart, at intervals of three to six weeks. ${ }^{49}$ All act by interfering with cell division, but of course they not only attack malignant cells but also depress the bone marrow, hair growth, and rapidly dividing cells in the gut. Side effects therefore occur frequently and may threaten life. Thrombocytopenia, with resultant spontaneous bleeding, is an uncommon but serious side effect, but nausea, vomiting, and alopecia are so common as to 
be expected in all patients. The peripheral blood count should thus be checked before the first dose of the drugs and a week to 10 days after these bolus doses (or three to four weeks after lomustine), when the white cell count will reach its nadir, but it recovers thereafter. This pancytopenia is also associated with varying degrees of immunosuppression, so that infections may also complicate treatment. ${ }^{50}$ None the less, many patients with small cell lung cancer can have a pain free life of reasonable quality considerably extended by these regimens at the cost of three or four days of anorexia and nausea every three or more weeks. Loss of hair is disguised by a wig. Most physicians think that the patient should know why such treatment is being recommended so that he may make an informed decision as to whether to accept this advice. Further information on new cytotoxic drugs is given elsewhere in this series.

\section{CYCLOPHOSPHAMIDE}

Cyclophosphamide, the well known alkylating agent, damages deoxyribonucleic acid (DNA) and so interferes with cell replication after hepatic metabolism that renders it active. Nearly all effective regimens for small cell cancer include cyclophosphamide, and in addition to the expected alopecia and pancytopenia (see above) this drug may also cause a distressing haemorrhagic cystitis from metabolites that accumulate in the bladder. This is common with regimens using an intravenous bolus of $2 \mathrm{~g}$ or more of cyclophosphamide, but symptoms of cystitis may develop in any patient with a disorder of the lower urinary tract. A high intake of fluid can minimise this but is not always effective. A new drug, mesna (mercaptoethane sulphonate), prevents cystitis by binding to the metabolites of cyclophosphamide in the urine, and so enables high doses (10-15 g) of cyclophosphamide to be given, without causing cystitis, in combination with autologous marrow transplantation. ${ }^{51}$ Mesna is not yet available on prescription in the United Kingdom.

\section{DOXORUBICIN}

Doxorubicin also interferes with DNA and RNA (ribonucleic acid) replication and produces complete alopecia as well as pancytopenia. It also increases sensitivity to therapeutic irradiation, given either before or afterwards. It is given intravenously into a fast running intravenous drip, as disastrous local reactions may follow extravasation into tissues. Prolonged administration may cause cardiomyopathy, but arrhythmias are commoner at even lower dosage. The total dose should not exceed $550 \mathrm{mg} / \mathrm{m}^{2}$ and should be less in patients with previous myocardial disease. ${ }^{52}$

\section{VINCRISTINE}

Vincristine is a plant alkaloid that is a useful adjunct to other chemotherapeutic regimens as it causes minimal marrow depression. A major side effect is neuropathy (sensory, motor, or autonomic), which is seen after long term administration. Extravasation into tissues from an intravenous infusion can cause local cellulitis.

\section{METHOTREXATE}

Methotrexate is an antimetabolite that inhibits dehydrofolate reductase and thus DNA synthesis. It has recently been given in high doses $\left(20-100 \mathrm{mg} / \mathrm{m}^{2}\right)$ intravenously followed after $12-24$ hours by rescue with folinic acid, which overcomes the enzymatic inhibition. Treatment, although effective, is potentially dangerous in patients with large pleural or peritoneal effusions, as the drug may later be released from these sites with subse- quent profound marrow depression. These high dose regimens, however, are thought to penetrate the central nervous system to treat micrometastases at that site. ${ }^{53}$

\section{LOMUSTINE}

Lomustine is a nitrosourea and alkylating agent (like cyclo- $\mathbb{D}$ phosphamide) that can also cross the blood brain barrier and 0 may be active in disease disseminated into the nervous system. Delayed suppression of the bone marrow may occur.

\section{ETOPOSIDE}

Etoposide is one of the most effective new agents against small cell lung cancer. ${ }^{54}$ It is usually given over three to four days every three to four weeks either by mouth or intravenously, and the. major side effects are alopecia, marrow depression, and gastrointestinal disturbances.

As with antituberculosis chemotherapy, such chemotherapy will usually be started by the respiratory physician or oncologist $\infty$ who has established the diagnosis of small cell cancer. As treatment may be prolonged, however, other doctors concerned with $\omega^{-}$ the patient's care during this time should have some knowledge 6 of the potential side effects of these chemotherapeutic regimens, of for these may well at first present to the patient's family doctor.

\section{References}

$(\mathbf{R}=$ Review article $)$

McHardy VJ, Inglis JM, Calder MA, et al. A study of infective and other factors in $\overrightarrow{c 0}$ exacerbations of chronic bronchitis. Br $\mathcal{F}$ Dis Chest 1980;74:228-38.

DEA. Comparison

Winston DJ, Lau WK, Gale RP Young LS. Trimethoprim-sulfamethoxazole for the treatment of pneumocystis carinii pneumonia. Ann Intern Med 1980;92. $762-9$

(IR) Wise R. Penicillins and cephalosporins: antimicrobial and pharmacological properties. Lancet $1982 ; \mathrm{ii}: 140-3$.

(1) Rolinson GN, Watson A, eds. Augmentin. Proceedings of the first symposium. Amsterdam: Excerpta Medica, 1981.

(1) Leigh DA, Robinson OPW, eds. Augmentin. Proceedings of the second symposium. $\overrightarrow{\bar{O}}$

Amsterdam: Excerpta Mal, Shaw EJ, Davies RJ. An assessment of the value of 3

Aumentin in the management of respiratory. An assessment of the value of 作 Excerpta Medica, 1982:138-49.

Philpott-Howard J, Williams JD. Increase in antibiotic resistance in Haemophilus influenzae in the United Kingdom since 1977: report of study group. $\mathrm{Br} \mathrm{Med} \mathcal{f}$ $1982 ; 284: 1597-9$.

British National Formulary No 4. London: British Medical Association and the Pharmaceutical Society of Great Britain, 1982.

作 , Wospital study of adult 3 . community-acquired pneumonia. Lancet $1982 ;$;ii: $255-8$. Edinburgh: Churchill Livingstone, 1981 .

(i) Neu HC. Clinical uses of cephalosporins. Lancet 1982;ii:252-5.

(K) Cameron IR, Phillips I. Pneumonia. In: Flenley DC, ed. Recent advances in Dene, 1980

${ }^{5}$ Monthly Index of Medical Specialities (MIMS). London: Medical Publications Ltd, N

'see GS, Kakati R, Mahmoud IA. A comparison of cefaclor and ampicillin in the I treatment of respiratory infection in elderly in-patients. Curr Med Res Opin 1980;O

6.564-8. bronchitis and pneumonia with cefaclor. Postgrad Med f $1979 ; 55$, suppl 4:59-61. MacKay AD. Amoxycillin versus ampicillin in treatment of exacerbations of chronic bronchitis. Br f Dis Chest 1980;74:379-84.

Havard CWH, Bax RP, Samanta TC, et al. Sputum and blood concentrations of $\omega$ cefuroxime in lower respiratory tract infection. Thorax $1980 ; 35: 379-83$. Cefotaxime symposium. Cefotaxime: a new cephalosporin antibiotic. I Antimicrob Chemother $1980 ; 6$, suppl A.

(1981;8, suppl B.

in

in severe respiratory tract infections. F Antimicrob Chemother 1982;9:479-84. compared in a double-blind study in the treatment of lower respiratory tract infections. Chemotherapy 1981;27:459-65.

" Nicotra MB, Rivera M, Awe RJ. Antibiotic therapy of acute exacerbations of chronic bronchitis. A controlled study using tetracycline. Ann Intern Med 1982; 97:18-2

Warren PM, Flenley DC, Millar JS, Avery A. Respiratory failure revisited: acute exacerbations of chronic bronchitis between 1961-8 and 1970-6. Lancet 1980;i:O

Warren PM, Jeffrey A, Haslett C, Wilkie RA, Flenley DC. Controlled oxygen therapy in acute exacerbations of chronic bronchitis and emphysema. Clin Scio 1982;63:53P.

Casewell MW, Talsania HG. Multiple antibiotic resistance and capsular types of 237-44.

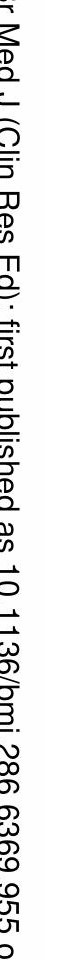




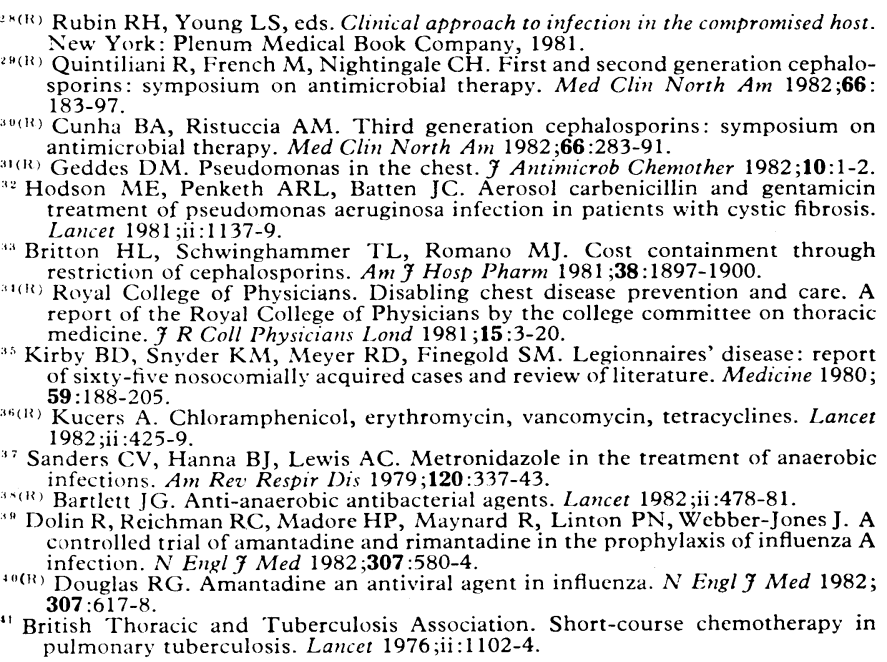

12 British Thoracic Association. Short-course chemotherapy in pulmonary tuber culosis. Lancet 1980;i:1182-3.

Nilsson BS, Boman G. Intravenous use of rifampicin. Eur $\mathcal{F}$ Respir Dis 1981 ;62: $212-4$

14 Medical Research Council Tuberculosis and Chest Disease Unit. National survey of tuberculosis notifications. in England and Wales 1978-9. Br Med $\mathcal{f} 1980 ; 281$ : 895-8.

45(R) Fox W. Short course chemotherapy for tuberculosis. In: Flenley DC, ed. Recent advances in respiratory medicine. II. Edinburgh: Churchill Livingstone,

${ }^{46}$ Angel JH. British Thoracic Association: a controlled trial of six months chemoin pulmonary tuberculosis. Am Rev Respir Dis 1982;126:460-2.

(11) Fox W. Whither short-course chemotherapy ? Br f Dis Chest 1981;75:331-57. (1) Sudlow MF. The treatment of lung cancer. In: Flenley DC, ed. Recent advances in respiratory medicine. II. Edinburgh: Churchill Livingstone, 1980.

Greco FA, Oldham RK. Current concepts in cancer: small-cell lung cancer. $N$ Engl f Med 1979:301:355-8.

s" Feld R. Complications in the treatment of small cell carcinoma of the lung. Cancer v $1981 ; 8: 5-25$.

Bryant BM, Jarman M, Ford HT, Smith IE. Prevention of isophosphamideinduced urothelial toxicity with 2-mercaptoethane sulphate sodium (mesnum) in patients with advanced carcinoma. Lancet 1980;ii:657-9.

(R) Young RE, Ozols RF, Myers CE. The anthracycline antineoplastic drugs. $N$ .

International symposium on methotrexate. Cancer Treat Rev 1981 ;65, suppl 1: $1-189$.

5s(R) Hansen HH, Rorth $M$. Lung cancer. In: Pinedo HM, ed. European Amsterdam: Excerpta Medica, 1980; 267-83.

\section{MATERIA NON MEDICA}

\section{Amateur naturalists and their occupation}

A serious interest in natural history is unusual even among countrymen, but its incidence has a noteworthy association with occupation.

The Victorian heyday of amateur naturalists, whose standards have seldom been equalled, was dominated by Anglican clergy and the medical profession.

No doubt because of the decreasing likelihood of discovering new species and the inhibiting effect of more professional naturalists, there was, at least in this county, a marked decline in the excellence of amateur naturalists during the first half of this century; but in the second half there has been a revival, stimulated partly by television, the profusion of superbly illustrated books, and the remarkable sophistication of the camera, leading to widespread rejuvenation of natural history societies by enabling lecturers to show slides which so beautifully reveal the intimate details of plant and animal life.

What, now, are the occupations of amateur naturalists ? Certainly they come from more diverse backgrounds, but the medical profession remains very much to the fore. Now that the profession is more specialised it might even be possible to correlate a love of natural history with different disciplines. My impression is that naturalists are to be found, at present, mainly among general practitioners and physicians, but seldom surgeons.

But how about the current neglect by the clergy ? Not just, I think, that they are dispirited by Darwinism. And why the lack of interest by lawyers-do they wrongly suppose that nature is chaotic?B E MILES, retired physician, Hereford.

\section{The age of the pak pai?}

In Hong Kong, with its overcrowded and inadequate roads, the "pak pai" is the answer to many businessmen's perennial problem of how to travel to and from work each day. The pak pai (or unlicensed taxi) is a hired vehicle whose legality is doubtful (due to lack of insurance cover for the occupants), whose mechanical condition would rarely pass an MOT inspection, and is driven by a young Chinese who is, euphemistically, eccentric.

The vehicles used are of two types-an elderly Mercedes, which is a vintage that will run for ever with minimal servicing, or a small cheap $1200 \mathrm{cc}$ Datsun van. The latter is usually yellow, has few if any springs and very hard seats. Being relatively new it doesn't need much servicing either.

The most prized benefit of a good pak pai is reliability. As the passengers number about five the pick up en route on crowded streets has to be a well co-ordinated operation literally timed to the minute. The knowledge that you will be unhesitatingly abandoned by being 30 seconds late in the morning is the ultimate spur for the tardy riser. In the evening the certainty that your transport will leave the office at a predetermined deadline is the incentive to ensure all meetings finish on time regardless of the agenda or the loquaciousnesc of other delegates. Many chairmen must envy this irreproachable reason for wielding the valedictory gavel. Wives approve of pak pais. Husbands rarely go drinking after office hours when the eventual return home involves an overcrowded bus or an expensive taxi, and the ladies have unrestricted access to the family vehicle five days per week.

The composition of this travelling caucus is an interesting exercise in group philosophy. Obviously everyone must live in the same area and work in the same district in the city to make a schedule feasible. This provides a multinational group and ensures that sport and politics are reviewed with a maximum of national bias and ferocious partisan attitudes. Only when criticising American football, which nobody understands, does the Commonwealth provide a united front.

The syndicate changes constantly because of transfer or repatriation, but browsing through old photographs-familiar faces but forgotten names-recalls many idiosyncratic personalities. Good humour and extreme scepticism are the sole common factors. One smiling face only appears in all the groups-Willie Cheong, the driver. Identifying the Yellow Peril we are, usually, referring to his Datsun pak pai.G D Ballantyne, Hong Kong.

\section{Don't call us, we'll call you}

Does anyone know a cure for telephonitis? Those of you with teenage children (especially girls-and I am being factual, not sexist) will know to what I am referring. Home from school 10 minutes and"I must phone Gloria." "You only left her half an hour ago." "Yes, but I forgot to ask about homework/netball/going out at the weekend," etc.

That's bad enough, but what about when they become students and develop friendships round the country? The old art of letter writing appears to be moribund and "having a chat" for 45 minutes over 200 miles even after $6 \mathrm{pm}$ is a lot more expensive than a stamp I will not dwell overlong on the situation when they start travelling. abroad, save to mention the afternoon (note afternoon) whon my elder daughter, who was working at Camp America, put in a reverse charge call to ask us to phone someone's parents in London to ask them to give a message to their son who was due to phone them from the USA, as there was no other way of contacting him! That added $£ 12$ to my account.

So what is the answer? One of my colleagues installed a pay phone in his daughter's bedroom as a birthday present, but when his bill arrived the following quarter it was as high as ever. "Have you been using my phone for your long distance calls ?" he asked. "Of course, Daddy," she replied sweetly. "I have to leave mine free for incoming calls, don't I ?"

I had an inspiration-a Telecom meter seemed to be the obvious solution, although $£ 5$ a quarter plus connection charge plus VAT seems a bit steep. But the only person who actually takes any notice of the spinning digits is me and it's my BP which suffers. I could throttle Buzby!-MYER GOLDMAN, radiologist, Liverpool. 